\title{
Prevalence of child sexual abuse: a comparison among 4 Italian epidemiological studies
}

\author{
Beatrice Castelli,1 Fiorella Festa,2 Maria Angela Di Sanzo,3 Andrea Guala,4 Alberto Pellai1 \\ 1Department of Biomedical Sciences, University of Milan; 2Department of Obstetrics and Gynecology, \\ SS Pietro e Paolo Hospital, Borgosesia (VC); 3 Tortora Rehabilitation Center, Local Health Unit 1, \\ Basilicata Region, Tortora; ${ }^{4}$ Department of Pediatrics, Castelli Hospital, Verbania, Italy
}

\begin{abstract}
Although many epidemiological studies defining child sexual abuse prevalence rates in many countries of the world are now available, Italy presents a lack of data regarding this specific issue. Only recently some attempts to define the epidemiology of this public health problem have been tried. Between 2003 and 2010, the Department of Public Health at University of Milan was in charge of one of the more important study of this kind in Italy, involving almost 3000 students aged 18 attending secondary schools in the City of Milan who filled out an anonymous questionnaire aiming at detecting their previous experiences of sexual victimization during childhood and adolescence. After this first edition, the study was replicated among students attending secondary schools in the city of Varese, in the area of Piedmont and in Basilicata (area of Lauria), using the same investigational model and questionnaire. This paper presents the main data collected through four different epidemiological studies using the same methodology and survey tool. Considering at least one of the five different forms of child sexual abuse surveyed (being exposed to pornography, being touched on private parts, being forced to masturbate an older person, being forced to perform oral sex, or being penetrated), the prevalence rate among our samples ranges between a minimum of $12.5 \%$ (Varese) and a maximum of $34.1 \%$ (Lauria). In Lauria the research reveals a higher prevalence rate of child sexual abuse, especially among the male population, for all typologies of abuse but being touched in their private parts. In all other geographical areas and for all typologies of abuse, there is always a higher prevalence among girls, with the only exception of abuse such as being exposed to pornographic materials.
\end{abstract}

Correspondence: Alberto Pellai, Department of Biomedical Sciences, University of Milan, via Pascal 36, 20133 Milan, Italy.

Tel: +39.02 .50315142 .

E-mail: alberto.pellai@unimi.it

Key words: Child sexual abuse; Epidemiology; Italy.

Received for publication: 16 July 2015.

Accepted for publication: 16 July 2015.

This work is licensed under a Creative Commons Attribution NonCommercial 3.0 License (CC BY-NC 3.0).

(C) Copyright B. Castelli et al., 2015

Licensee PAGEPress, Italy

La Pediatria Medica e Chirurgica 2015; 37:114

doi:10.4081/pmc.2015.114

\section{Introduzione}

\section{Epidemiologia del fenomeno a livello internazionale}

Gli abusi sessuali sono sempre esistiti ma solo nel corso del XX secolo il crescente aumento dell'attenzione rivolto all'infanzia e alla sua tutela ha consentito una progressiva consapevolezza della gravità della diffusione del fenomeno dell'abuso sui bambini. Questo fenomeno ha richiesto molto tempo ed impegno per essere conosciuto e riconosciuto come un problema sociale; continua però a rimanere sottostimato. È difficile stabilire quando l'abuso sessuale ha fatto la sua apparizione nella società occidentale e quando ha iniziato a essere percepito come un problema.

Le prime ricerche sugli abusi sessuali sui minori sono state condotte soltanto negli ultimi decenni; negli USA questo problema ha cominciato ad essere reso pubblico già verso la fine degli anni '70. Fino allora, dato lo scarso numero di casi di abuso sessuale riportati, si pensava che il problema fosse poco rilevante. Finkelhor, un ricercatore americano che da molti anni si occupa dello studio dell'abuso sessuale, ha paragonato 20 studi epidemiologici condotti al di fuori del NordAmerica. 1,2 Le ricerche sono state condotte nel Nord Europa e in paesi di lingua inglese; sono stati anche analizzati alcuni studi effettuati in tre paesi di lingua spagnola, Costa Rica, ${ }^{3}$ Repubblica dominicana, ${ }^{4}$ Spagna, ${ }^{5}$ e in Grecia. ${ }^{6}$ Queste ricerche variano molto negli obiettivi, nel campione selezionato e negli strumenti utilizzati. Tutti gli studi disponibili hanno identificato una storia di abuso sessuale in almeno il $7 \%$ delle donne e il $3 \%$ degli uomini fino a una percentuale pari al $36 \%$ nelle donne in Austria e 29\% negli uomini in Sud Africa. ${ }^{7}$

Una ricerca condotta da Muhammad nel 2001 in Palestina, ${ }^{8}$ con lo scopo di determinare il tasso di prevalenza di abusi tra gli studenti universitari è arrivata alla conclusione che il 18,6\%, il 36,2\% e il 45,6\% degli studenti intervistati afferma di essere stato vittima durante l'infanzia rispettivamente da parte di un membro della famiglia, di un parente e di uno sconosciuto. Recentemente è stato pubblicato uno studio condotto in 4 province della Cina $^{9}$ con un campione molto numeroso: 2300 studenti delle scuole superiori. L'analisi dei dati evidenzia che il 16,7\% delle femmine e il 10,5\% dei maschi ha subito un abuso sessuale prima dell'età di sedici anni, dato che è poi stato correlato con un maggior rischio di abuso di alcool, suicidi, umore depresso, e disturbi del comportamento alimentare (solo nelle femmine). Lo studio ha evidenziato che il rischio associato al fenomeno dell'abuso non è correlabile con la dimensione della famiglia, l'educazione ricevuta 0 con l'ambiente rurale 0 urbano in cui è vissuto l'adolescente. Infine, benché inseriti in un contesto socio-culturale molto diverso da quello occidentale, è emerso che il profilo psicologico e comportamentale dei giovani cinesi che hanno subito un abuso sessuale infantile è del tutto simile a quello riscontrato in altre culture. 
Nonostante le diverse metodologie utilizzate e lo strumento adottato, tutti gli studi suggeriscono che l'abuso sessuale è un problema a livello internazionale e trasversale in tutte le società, occidentali e orientali, e che è molto più diffuso di quanto ci suggeriscono i casi riportati alle autorità locali. Non ci sono indicatori che permettono di escludere la possibilità di abuso sessuale in un paese 0 in un altro, né si può determinare a priori quali sono i bambini che per caratteristiche di età, sesso o background culturale siano a minor o maggior rischio rispetto ad altri. Per rispondere alla domanda su come variabili sociali e culturali possono essere associati al rischio di abuso sessuale, sono necessari ulteriori studi, più raffinati dal punto di vista metodologico. Un limite degli studi sopra analizzati è che gli eventi accaduti durante l'infanzia e l'adolescenza si basano sui ricordi dell' adulto. In particolare, i racconti di esperienze subite nell'infanzia e adolescenza possono essere soggetti a fallibilità dovuta a problemi legati al recall mnemonico, interpretazione distorta delle esperienze provate, riluttanza da parte della vittima a rivelare tali esperienze e infine incapacità di ricordare eventi dolorosi. ${ }^{10}$ I ricercatori dovrebbero costruire strumenti di confronto internazionale, selezionare definizioni comuni dell'AS e infine utilizzare questionari con domande molto precise sui diversi aspetti dell'abuso sessuale.

\section{Epidemiologia del fenomeno in Italia}

In Italia il fenomeno è molto meno conosciuto e definito rispetto agli altri paesi: gli unici dati a nostra disposizione sono le denunce di abuso registrate al Tribunale di Grazia e Giustizia. Negli ultimi 15 anni si è assistito ad un incremento degli abusi denunciati che denota sicuramente una maggiore visibilità del fenomeno; tuttavia il fenomeno è da considerarsi sottostimato. Considerata la scarsità degli studi di prevalenza in Italia, l'allora Istituto di Igiene e Medicina Preventiva dell'Università degli Studi di Milano, poi divenuto Dipartimento di Sanità Pubblica dell'Università degli Studi di Milano, in collaborazione con il Servizio Famiglia Infanzia della ASL Città di Milano, ha deciso di implementare uno studio ad hoc per indagare la prevalenza di abuso sessuale tra gli studenti diciottenni dell'ultimo anno delle scuole medie superiori. ${ }^{11}$ In Italia un'altra ricerca, condotta in Veneto, ha tentato di stimare il fenomeno; 12 quest'ultimo studio ha coinvolto 638 studenti diciottenni ai quali è stato fatto compilare un questionario anonimo. Gli autori evidenziano che il $24,4 \%$ delle femmine, (il $10,8 \%$ in modo grave e il $13,6 \%$ in modo lieve), e il 14,2\% dei maschi (il 2,8\% in modo grave e l'11,4\% in modo lieve) ha dichiarato di essere stato vittima di abuso sessuale durante l'infanzia.

Complessivamente, considerando tutte le forme di abuso indagate (essere esposti alla visione di materiale pornografico, essere toccato in parti intime da parte di una persona più grande, essere costretto a toccare i genitali di una persona più grande, essere costretto a un rapporto orale 0 alla penetrazione da parte di una persona più grande) il 14,6\% ha dichiarato di aver subito almeno una delle tipologie di abuso sopra elencate. Questa percentuale può essere poi scorporata in due componenti: il $12,3 \%$ dichiara di aver subito un abuso che è stato classificato come lieve-moderato e comprende le prime tre tipologie, mentre l'abuso grave è stato subito dal $2,3 \%$ degli studenti. Se comprendessimo anche i casi di ragazzi che hanno risposto preferisco non rispondere, le percentuali aumenterebbero dal $14,6 \%$ al 15,4\%. La probabilità di essere vittimizzate è maggiore nelle ragazze rispetto ai ragazzi per tutte le tipologie di abuso tranne l'esposizione alla visione di materiale pornografico.

I risultati sopra descritti hanno senza dubbio portato alla luce un fenomeno che troppo a lungo si è voluto negare e tenere nascosto e che, grazie alla collaborazione e alla sincerità dei ragazzi, si è potuto analizzare in tutta la sua crudezza. È naturale tuttavia domandarsi se questi dati possano realmente rappresentare la realtà italiana o solo la realtà isolata di una grande città come Milano. Al fine di confermare tali risultati e raccogliere ulteriori esperienze lo studio è stato replicato nella provincia di Varese durante l'anno scolastico 2004-2005. Dallo studio emerge che, nel campione, costituito da 561 studenti, il 12,5\% ha dichiarato di aver subito una delle cinque tipologie di abuso indagate e che l'abuso sessuale rappresenta un fattore predisponente per l'adozione di comportamenti patologici verso il cibo indagati tramite l'Eating Attitude Test e la compilazione delle figure di Collins.

Un altro studio molto interessante è stato compiuto su un campione molto vasto, 1300 studenti frequentanti il quinto anno di scuola superiore del comune e provincia di Vicenza nel 2005.13 È stata dedicata un'ora per la presentazione della ricerca e della problematica in tutti i suoi aspetti, due settimane dopo gli operatori hanno fatto compilare, in classe, il questionario. Il questionario, anonimo, è composto da 20 domande, divise in 5 parti; lo studio conclude che il $31 \%$ degli studenti ha subito violenza, di questi poco più della metà (17\%) ha subito violenza sessuale (13\% con contatto), l'11\% del campione ha subito violenza psicologica, mentre il $3 \%$ del campione ha subito violenza fisica.

È stato recentemente pubblicato un testo ${ }^{14}$ che raccoglie un'indagine epidemiologica condotta tra luglio 2004 e febbraio 2005 in sei regioni italiane (Lombardia, Friuli Venezia Giulia, Toscana, Umbria, Puglia e Calabria), rappresentative di tutto il territorio, su un campione di 2320 donne di età compresa tra i 19 e 60 anni. La metodologia utilizzata è stata quella dell'intervista telefonica e dell'intervista faccia a faccia mediante l'ausilio di questionari già predisposti, di tipo semi strutturato, costituiti da domande chiuse, semi aperte ed aperte. I soggetti intervistati sono stati raggruppati in: coloro che non riferiscono alcuna forma di abuso sessuale 0 maltrattamento, coloro che riferiscono di aver subito una forma di abuso sessuale, donne che riportano maltrattamenti intrafamiliari e donne che denunciano sia maltrattamenti che abusi sessuali. L'abuso sessuale è stato riferito dal 5,9\% del campione. La forma prevalente è risultata l'esposizione a materiale pornografico e l'esibizionismo, pari al $64 \%$ di tutte le forme riferite. Il $34 \%$ riporta forme di abuso con contatto fisico (essere toccate, atti di masturbazione, tentativi di penetrazione) mentre l'1,6\% riferisce abuso sessuale con penetrazione. L'indagine mostra che l'abuso viene rilevato da circa il $60 \%$ delle vittime, ma in misura maggiore da chi ha subito un abuso lieve. Purtroppo alla confidenza spesso non è seguita un'azione: solo in un terzo dei casi rivelati a qualcuno sono discesi fatti concreti. La ricerca inoltre correla la pregressa vittimizzazione sessuale con disturbi psichici e somatici, in particolare evidenzia che il $23,6 \%$ delle vittime è affetto da disturbi del comportamento alimentare e più della metà soffre di depressione.

\section{Obiettivi delle ricerche}

Considerati i risultati delle ricerche effettuate a Milano e Varese che hanno confermato quanto l'abuso sessuale sia un problema presente anche in Italia, l'Istituo di Igiene e Medicina Preventiva dell'Università degli Studi di Milano ha deciso di allargare i territori d'indagine così da validare ulteriormente i risultati ottenuti. Gli obiettivi delle ricerche che saranno esposte sono: i) definire il tasso di prevalenza di vittimizzazione sessuale all'interno della coorte degli studenti frequentanti le $\mathrm{V}$ classi delle scuole superiori presenti nella città di Vercelli, Borgosesia (VC) e Lauria (PZ) durante l'anno accademico 2005-2006; ii) definire le caratteristiche degli episodi di vittimizzazione sessuale subite in infanzia dalle vittime relativamente a questi parametri: natura dell'abuso, tipologia dell'abusante, età della vittima al momento dell'abuso, rivelamento dell'abuso ad altra persona fidata, eventuale intervento di operatori professionisti a sostegno della vittima; iii) confrontare quanto rilevato dagli studi effettuati in Piemonte e in Basilicata con quanto emerso nelle ricerche precedenti (Varese e Milano).

\section{Materiali e Metodi}

Le ricerche che sono qui presentate sono state configurate come studi epidemiologici retrospettivi realizzati attraverso la somministrazione di questionari anonimi a un campione degli studenti frequentan- 
ti la quinta classe delle scuole superiori presenti nelle città di Vercelli, di Borgosesia e Lauria. La metodologia della ricerca ha previsto le fasi di lavoro sotto descritte.

\section{L'organizzazione dello studio Fase I}

Proposta del progetto, secondo il protocollo utilizzato per le ricerche effettuate a Milano e Varese, alle scuole selezionate.

\section{Fase II}

Programmazione dello studio con identificazione delle classi $\mathrm{V}$ da coinvolgere, messa a punto della metodologia di somministrazione, e raccolta dei questionari compilati. Nella selezione delle classi da coinvolgere si è cercato di selezionare un campione di scuole che fosse rappresentativo dei diversi indirizzi e tipologie di scuole superiori presenti sul territorio della Provincia.

\section{Fase III}

Formazione dei somministratori: medici dell'Istituto di Igiene e Medicina Preventiva di Milano hanno formato gli operatori dell'ASL/Ospedale di Vercelli e Borgosesia approfondendo la metodologia utilizzata per la somministrazione e il ritiro dei questionari ed evidenziando le potenziali criticità legate alla ricerca come per esempio la scarsa partecipazione degli insegnanti. La distanza con Lauria non ha permesso lo stesso percorso con gli operatori della Basilicata che hanno lavorato più autonomamente.

\section{Fase IV}

Somministrazione, raccolta e invio al gruppo di ricerca dei questionari compilati dagli adolescenti.

\section{Fase $V$}

Caricamento su sistema informatico (EPIDATA) di tutti i questionari raccolti e conseguente analisi statistica dei dati tramite SPSS.

\section{Lo strumento d'indagine}

Considerate le esperienze delle precedenti ricerche e per un miglior confronto dei dati si è ritenuto di poter utilizzare il medesimo questionario adoperato per gli studi effettuati in Lombardia. Lo strumento consta di una sezione introduttiva mentre il vero e proprio questionario è suddiviso in tre parti: i) la presentazione è finalizzata a motivare il rispondente, spiegare lo scopo dello studio e le modalità di compilazione, fornire le garanzie di anonimato e privatezza; ii) la prima parte del questionario punta ad indagare le eventuali esperienze di pregresso abuso, oltre a raccogliere informazioni socio-demografiche, informazioni sulla sessualità e informazioni relative alla eventuale partecipazione ad attività di informazione e prevenzione primaria relative all'abuso; iii) la seconda parte indaga, tramite il test delle figure di Collins, la percezione del proprio corpo che hanno gli adolescenti intervistati, raccogliendone inoltre i dati antropometrici; iv) la terza parte è costituita dal test EAT 26, finalizzato a rilevare la predisposizione ai disturbi del comportamento alimentare.

\section{Procedura di selezione del campione}

In considerazione della natura della ricerca, sono stati coinvolti soltanto studenti maggiorenni frequentanti le classi quinte, poiché in grado di autodeterminare la propria partecipazione allo studio senza alcun bisogno di altra autorizzazione. I dirigenti delle scuole superiori della Provincia sono stati informati dello studio e a essi è stato richiesto il consenso e l'autorizzazione allo svolgimento della ricerca all'interno della propria scuola secondo il protocollo di ricerca preparato ad hoc.

\section{Modalità di somministrazione del questionario}

La somministrazione del questionario, in ciascuna delle scuole coinvolte, è stata eseguita all'interno delle singole classi, alla presenza di personale opportunamente addestrato e non appartenente al corpo docente. Il questionario è stato presentato e consegnato ai ragazzi nel corso di una mattinata scolastica da un operatore esterno alla scuola. A differenza di quanto avvenuto nelle precedenti ricerche, gli operatori dell'ASL di Borgosesia, considerato l'esiguo numero di persone a disposizione e la densità abitativa, hanno ritenuto, al fine di ottimizzare le risorse, più opportuno lasciare in ognuna delle classi una scatola chiusa dove i ragazzi potessero depositare il questionario sigillato in una busta. La scatola è stata ritirata dagli stessi operatori dopo circa una settimana dal momento della presentazione della ricerca. È importante qui rilevare che la scelta di ritirare il questionario il giorno successivo alla consegna, è stata dettata, nelle precedenti ricerche, da precise esigenze, quali garantire l'assoluto anonimato dei ragazzi. Inoltre, premeva che i ragazzi compilassero il questionario in totale solitudine, possibilmente a casa, in un momento da loro scelto. La totale sincerità dei ragazzi e la completezza dei questionari hanno fornito sufficienti prove della correttezza di tale accorgimento. Il questionario compilato e chiuso in una busta è stato poi inviato al gruppo di ricerca centrale per l'elaborazione e l'analisi dei dati.

\section{Risultati}

Verranno di seguito descritte le caratteristiche principali relative alle due più recenti ricerche, quella condotta in Piemonte e quella condotta in Basilicata.

\section{Piemonte}

Sono stati raccolti e informatizzati 388 questionari, pari al 90\% di quelli consegnati (430). Solo 5 (pari all'1,2\%) sono i questionari non ritenuti validi, pertanto esclusi dalla presente trattazione. Gli Istituti scolastici coinvolti sono complessivamente 12 , con diverso indirizzo di studio comprendendo licei, istituti tecnici e professionali.

Il campione intervistato presenta una leggera prevalenza di maschi rispetto alle femmine (rispettivamente $53,1 \%$ contro 46,9\%). L'età dei ragazzi è in maggioranza compresa tra 18 e 19 anni, infatti l'età media dei partecipanti è risultata di 18,4 anni, equivalente nei maschi e nelle femmine.

Alcune domande miravano a verificare quanto i ragazzi sono contenti di come sono (poco più della metà lo è) e approfondire le relazioni interpersonali. Da qui emerge che circa il $7 \%$ ritiene i propri rapporti al di fuori della famiglia poco o per nulla soddisfacenti.

Alla domanda se avessero già avuto un rapporto sessuale completo il $61,3 \%$ degli studenti intervistati ha risposto affermativamente, lievemente di più le femmine $(63,7 \%)$ rispetto ai maschi $(59,2 \%)$. Un ragazzo su quattro aveva più di 17 anni, mentre il partner aveva mediamente venti anni. La percentuale aumenta riguardo i rapporti sessuali parziali: complessivamente il $77,6 \%$ ha risposto affermativamente a tale domanda. Una delle domande del questionario chiedeva se avessero mai avuto rapporti prima dei 14 anni con un/una partner di età superiore ai 17: circa il 5\% ha risposto affermativamente. Alla domanda se avessero mai avuto una relazione stabile ha risposto affermativamente il 76,5\% dei ragazzi: la durata della relazione è stata variabile tra un mese e un anno ma quasi un ragazzo su cinque ha dichiarato di aver vissuto un rapporto più lungo di due anni. Il $65 \%$ degli studenti è stato molto/abbastanza soddisfatto della propria relazione. La fine della storia è stata decisa dal ragazzo stesso nel $22 \%$ dei casi mentre ha subito la decisione il 16\% di loro. Diversi sono i sentimenti che hanno accompagnato la fine della relazione, equamente distribuiti tra il senso di 
liberazione, di colpa, il distacco emotivo, o in alcuni casi anche con un senso di disperazione.

Uno degli obiettivi della ricerca era quello di approfondire le conoscenze e i variegati aspetti dell'abuso sessuale all'infanzia così da migliorare i progetti di prevenzione primaria già implementati. Sorprende ancora che a più della metà di questi ragazzi quando erano bambini nessun adulto abbia mai spiegato cosa è l'AS, in particolare ai maschi (59,7\% vs 48,9\% delle femmine); complessivamente il $37 \%$ di essi, sempre con differenze tra maschi e femmine, ha ricevuto informazioni dai genitori mentre risulta ancora troppo carente il messaggio che proviene dalla scuola (13\%). Sono sempre tanti (circa il 50\%) i ragazzi che dichiarano di non aver ricevuto informazioni su come difendersi in situazioni di rischio e, analogamente alla domanda precedente sembra che si presti maggior attenzione alle femmine, le più deboli, indifese e maggiormente vittime rispetto ai coetanei convinti che il maschio non possa subire maltrattamenti e abusi.

\section{Lauria}

Sono stati raccolti e informatizzati 123 questionari, pari al $73,6 \%$ di quelli consegnati (167). La percentuale di ritorno rappresenta ancora un buon indicatore dell'attività svolta ma è lievemente inferiore rispetto a quanto emerso nelle precedenti ricerche. Gli Istituti scolastici coinvolti sono complessivamente 3 , con diverso indirizzo di studio e comprendono un liceo classico, un IPSIA ed un IPC.

Il campione intervistato presenta una prevalenza di maschi rispetto alle femmine (rispettivamente $57,7 \%$ contro $40,7 \%$ ). L'età dei ragazzi è in maggioranza compresa tra 18 e 19 anni, infatti l'età media dei partecipanti è risultata di 18,2 anni, equivalente nei maschi e nelle femmine. Dall'analisi emerge che solo il $4 \%$ dei ragazzi è figlio unico, e la maggior parte (92\%) vive con entrambi i genitori. I ragazzi dichiarano di essere piuttosto soddisfatti delle proprie relazioni con persone al di fuori della famiglia e solo l'8\% non è molto contento.

Alcune domande indagavano la vita sessuale dei ragazzi: 4 ragazzi su 10 hanno affermato di aver avuto rapporti sessuali completi, il 52,1\% dei maschi ed il 34\% delle femmine. L'età del primo rapporto si colloca mediamente per il 20\% dopo i 17 anni e per il 12\% dopo i 16 anni ma per il 25\% dei maschi l'età risulta compresa tra i 14 e i 15 anni mentre il partner delle ragazze aveva mediamente 19 anni e mezzo e la partner dei ragazzi 16 anni e mezzo. La percentuale aumenta relativamente ai rapporti sessuali parziali: complessivamente il $63,4 \%$ ha risposto affermativamente a tale domanda.

Una delle domande del questionario chiedeva se avessero mai avuto rapporti prima dei 14 anni con un/una partner di età superiore ai 17: circa l' $11 \%$ ha risposto affermativamente, il $6 \%$ delle femmine e il $14 \%$ dei maschi. Relativamente ad un rapporto sentimentale stabile, il $30 \%$ dei ragazzi afferma di non aver mai vissuto una storia; tra chi ha risposto di sì a tale domanda, più della metà $(56 \%)$ lo ha giudicato soddisfacente 0 abbastanza soddisfacente. La fine della storia è avvenuta per diversi motivi o perché sono stati lasciati dal/dalla partner $(14,6 \%)$, nel $16,3 \%$ dei casi hanno deciso di interrompere la relazione oppure nel $17 \%$ delle storie si sono lasciati di comune accordo. La fine del rapporto è vissuta in un caso su cinque con un senso di distacco emotivo, anche se il $10 \%$ dei ragazzi ha dichiarato di aver provato un senso di liberazione; pochi invece (6) hanno affermato di aver provato un senso di abbandono e di colpa.

\section{Discussione}

Uno degli obiettivi della ricerca è di approfondire le conoscenze e i variegati aspetti dell'abuso sessuale all'infanzia così da migliorare i progetti di prevenzione primaria già implementati. Più della metà dei ragazzi (57\%) ha affermato di non aver mai ricevuto informazioni su cosa è l'abuso sessuale, in particolare la prevalenza è più alta nelle femmine (64\%) rispetto ai maschi (53\%) a differenza di quanto evidenziato nelle precedenti ricerche. Le spiegazioni, quando presenti, provengono prevalentemente dai genitori (25\%) mentre è ancora troppo carente il messaggio fornito dalla scuola $(14,6 \%)$. Sono sempre tanti (circa il 50\%) i ragazzi che dichiarano di non aver ricevuto informazioni su come difendersi in situazioni di rischio, in particolare le femmine (56\%). Verranno di seguito confrontati i risultati delle quattro ricerche effettuate relative alle singole tipologie di abuso precedentemente esposte.

\section{Esposizione a materiale pornografico}

La Figura 1 mostra che in tutte e quattro le ricerche, la prevalenza di questa tipologia di abuso è maggiore nei maschi rispetto alle femmine. Appare abbastanza uniforme il dato emerso per le femmine nei campioni di Milano, Varese e Lauria mentre risulta decisamente superiore in Piemonte; inoltre, si osserva che in quest'ultimo caso e a Lauria la prevalenza nei maschi è decisamente superiore rispetto alle ricerche precedenti. La frequenza di questa tipologia di abuso oscilla da un minimo del $2 \%$ a un massimo del $12,6 \%$ per le femmine mentre per i ragazzi l'intervallo è compreso tra $4,1 \%$ e $18,3 \%$. Si conferma il rapporto di conoscenza tra vittima e abusante: la persona che ha agito l'abuso era infatti ben conosciuta in ogni ricerca, con una frequenza compresa tra 60 e 70\%. Quando è stato specificato si è trattato più spesso di un parente stretto (zio, cugino) 0 di un amico. L'età media in cui si è verificato il primo episodio è compresa tra 10 e 13 anni, ed è risultata sempre superiore nei ragazzi rispetto alle ragazze. È stato riscontrato che alle vittime è stato imposto il segreto in un terzo dei casi nei campioni di Milano e Varese, ai 2/3 delle vittime di Lauria, mentre risulta inferiore la percentuale delle vittime piemontesi che ha riferito di essere stato obbligato a non raccontare a nessuno l'accaduto (16,7\%). Il dato risulta superiore per le femmine rispetto ai maschi ad eccezione della Basilicata. Inoltre, mentre nelle ricerche effettuate in Lombardia la percentuale dei ragazzi che è riuscito a confidarsi con qualcuno è poco inferiore al 50\%, e le ragazze decidono di farlo più frequentemente, nelle ricerche più recenti i $2 / 3$ dei ragazzi hanno in effetti tenuto per sé l'accaduto. In ogni caso, quando le vittime disvelano l'abuso subito, lo fanno prevalentemente a un amico, come dimostra anche la scarsità di ragazzi che hanno ricevuto aiuto da parte di esperti (il $4 \%$ circa delle vittime lombarde, una vittima piemontese, nessuna vittima di Lauria).

\section{Toccato in parti intime}

Ricordano di essere stati toccati in parti intime in un modo che ha creato disagio il $6,4 \%$, il 7,3\%, l'8,9\% e l'11,3\% rispettivamente dei

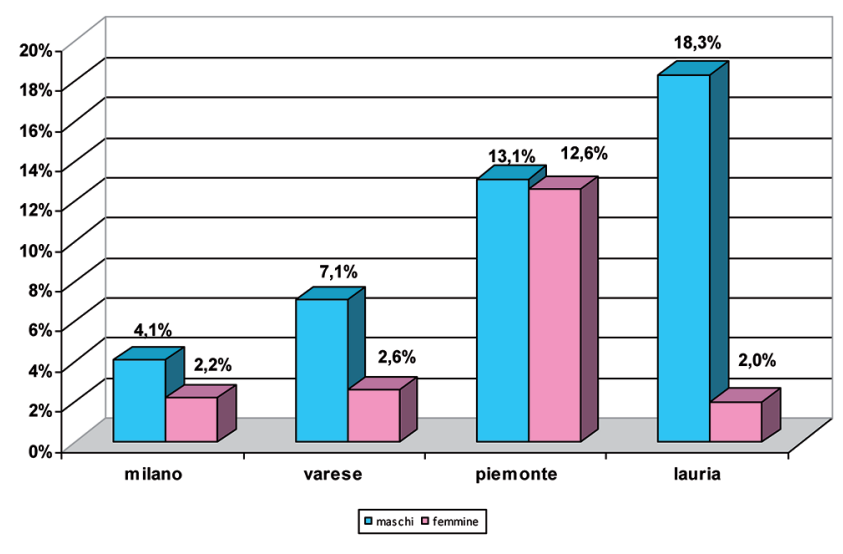

Figura 1. Essere esposti a materiale pornografico 
ragazzi intervistati in Piemonte, a Varese, Lauria e Milano. La Figura 2 mostra che questa tipologia di abuso colpisce più frequentemente le ragazze rispetto ai ragazzi: nonostante i dati siano uniformi nei quattro studi, si è rilevato che a Milano circa quattro ragazze per classe hanno risposto affermativamente a questa domanda. Una grande città come il capoluogo lombardo, dove frequenti sono gli episodi sui trasporti pubblici, potrebbe in effetti giustificare un dato sproporzionato rispetto ai restanti campioni. La metà delle vittime riporta di conoscere l'abusante (tutte le vittime di sesso femminile intervistate a Lauria) e quando specificato, è indicato in un parente appartenente alla famiglia allargata (zii, cugini, ecc...) dal 40\% delle vittime lombarde, e dal $20 \%$ dei restanti campioni. L'età media in cui è accaduto l'episodio è stabile tra i 12 e i 13 anni, sempre più alta per i maschi rispetto alle femmine. Relativamente all'imposizione del segreto da parte dell'abusante alla propria vittima, ha risposto affermativamente il $64 \%$ delle vittime della Basilicata rispetto ad una percentuale variabile tra il 16 e il $25 \%$ nelle altre ricerche. Hanno riferito a qualcuno l'episodio: il 50\% di coloro che l'hanno subito in Piemonte, il 60\% delle vittime di Lauria e 75\% a Milano e Varese. Quando è stato approfondito quest'aspetto si è rilevato che i ragazzi si sono confidati molto poco con adulti, soprattutto i maschi. Si conferma molto bassa (tra il 5 e il 7\%) la percentuale di coloro che hanno ricevuto aiuto da parte di esperti.

\section{Costretto a toccare}

Riguardo l'essere stati costretti a toccare i genitali di persone adulte, le nostre ricerche forniscono dati omogenei, in particolare la prevalenza è compresa tra $3,1 \%$ (Varese) e $3,2 \%$ (Lauria) (Figura 3 ). Nello specifico, ciò che differenzia quest'ultimo campione dalle altre ricerche, dove le femmine erano le più colpite, è l'assenza di casi riportati dalle ragazze. L'abusante era ben conosciuto dalle vittime in tutti gli studi, ad eccezione del campione della Basilicata, dove gli abusi sono stati agiti da persone sconosciute nella metà dei casi. Quando è stato specificato, l'abusante è identificato in un membro della famiglia allargata (cugino/a, zio), in un amico mentre un ragazzo di Lauria indica il padre come responsabile dell'abuso.

L'età del primo episodio è pari a 11,5 nelle ricerche di Milano, Varese e Piemonte mentre è superiore nelle vittime di Lauria. In alcuni casi (es. Piemonte) l'età è superiore nelle femmine rispetto ai maschi, il contrario si è rilevato in altri studi (Milano e Varese).

L'imposizione del segreto è atto molto frequente, in particolare al Sud: infatti le vittime che dichiarano di essere state costrette a tenere per sé l'accaduto sono il 33,8\%, il 58,3\%, il 62,5\% e l'83,3\% rispettivamente nelle ricerche di Milano, Varese, Piemonte e Lauria, e in effetti hanno riferito l'episodio tra il 35 e il $50 \%$ delle vittime, ma ancora più esiguo è il numero di coloro che si sono confidati con adulti. Il tempo medio intercorso tra l'episodio e la prima volta in cui è stato riferito è mediamente di oltre due anni. Nessuna tra le vittime del Piemonte o della Basilicata ha ricevuto aiuto da un esperto dopo quanto accaduto, mentre i ragazzi che sono stati supportati da personale esperto sono il 9,5\% a Milano e l'8,3\% a Varese.

\section{Costretto a masturbare}

Ricordano di essere stati obbligati a masturbare persone adulte 0 più grandi lo $0,7 \%$, l'1,4\%, l'1,6\% e il 2,1\% rispettivamente dei ragazzi di Varese, Milano, Piemonte e Lauria (Figura 4). I dati rilevati confermano ulteriormente la validità dello strumento e delle metodologie utilizzate, nonché l'attendibilità di quanto riferito dai ragazzi. Analizzando la distribuzione per sesso si osserva che questa tipologia di abuso è prevalente nelle femmine in 3 dei campioni studiati, in particolare Milano, Piemonte e Varese; in quest'ultimo caso peraltro nessun maschio ha risposto affermativamente mentre a Lauria è stata riportata maggiormente dai ragazzi $(2,0 \%$ vs $1,4 \%)$. L'abusante era conosciuto da tutte le vittime di Milano e Varese e identificato con il partner o expartner per il $60 \%$ di esse e nel 30\% identificato con un familiare. Per quanto riguarda il Piemonte, nel $75 \%$ dei casi si è trattato di una persona conosciuta dalla vittima, in due casi (25\%) si è trattato di un parente stretto (cugino/zio), una ragazza ha dichiarato che si è trattato del fidanzato, mentre l'abusante è stato identificato in un amico da parte di tre vittime (37,5\%). Nello studio della Basilicata la ragazza ha riferito di essere stata costretta a subire l'abuso da più persone (non specificate) mentre il ragazzo ha riferito che si trattava del padre. L'età della vittima in cui è avvenuto l'episodio per la prima volta è compresa tra 12 e 14 anni.

Relativamente all'imposizione del segreto, vi è una certa variabilità, si passa infatti dal $25 \%$ delle vittime di Varese al $62,5 \%$ delle vittime Piemontesi che riferiscono di essere state costrette a non riferire l'accaduto. Dallo studio infatti emerge che le vittime del Piemonte e di Lauria sono quelle che maggiormente hanno tenuto per sé il segreto: sono 5 ed una rispettivamente le vittime che si sono confidate, mentre il $60 \%$ delle vittime milanesi e il $2,8 \%$ delle vittime di Varese ha riportato l'accaduto. Infatti, il 15\% delle vittime del capoluogo lombardo ha ricevuto aiuto da parte di personale specializzato in relazione all'episodio, una sola vittima piemontese e nessuna nelle altre due ricerche.

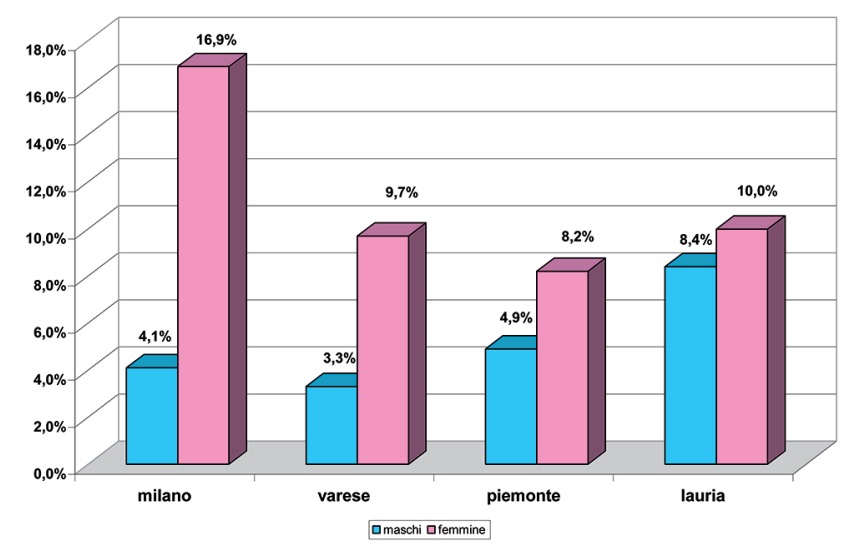

Figura 2. Essere toccati nelle proprie parti intime

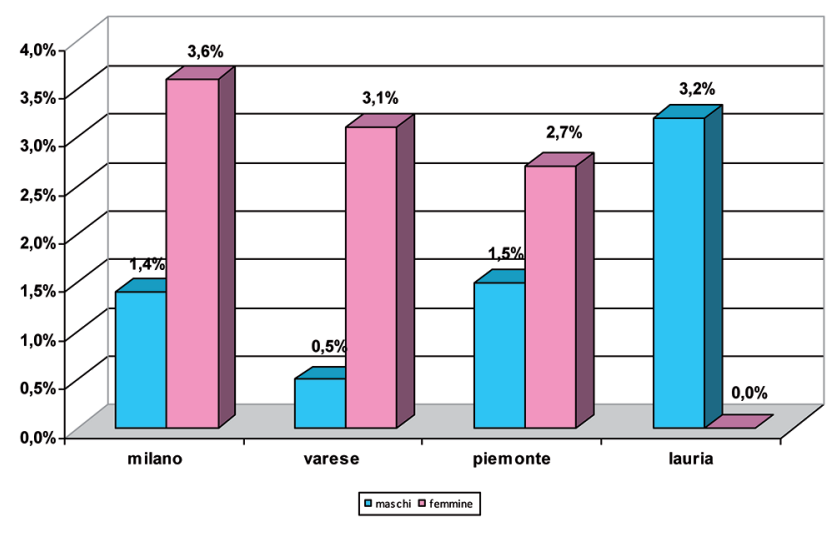

Figura 3. Essere costretti a toccare le parti intime di un altro/a. 


\section{Penetrazione}

La Figura 5 illustra che relativamente alla domanda ricordi ancora oggi che ti sia capitato che qualche adulto ti abbia obbligato ad una penetrazione (anale, vaginale o orale) o ha tentato di farlo si è riscontrata una prevalenza superiore nelle femmine nelle ricerche condotte a Milano e Varese mentre negli studi Piemontese e Lucano vi è una inversione dei risultati. In particolare, si evidenzia che la prevalenza complessiva è compresa tra $1,1 \%$ e $4,8 \%$ e che la frequenza più alta è emersa in Basilicata, accompagnata da una decisa predominanza nel sesso maschile.

Relativamente alla persona che ha commesso l'abuso, le vittime, in particolar modo le ragazze, hanno dichiarato di conoscerla bene in una percentuale tra il 50 e il 60 , nello specifico si è trattato prevalentemente di un membro della famiglia allargata (zio, cugino), amici o nel caso di Milano più della metà delle vittime ha identificato il responsabile nel partner 0 ex partner. L'età media della vittima all'epoca dell'abuso risulta compresa tra 9 anni (Lauria) e 15 anni (Milano), generalmente superiore per i maschi rispetto alle femmine.

Labusante ha imposto di mantenere il segreto rispettivamente al $70 \%, 60 \%$, e $30 \%$ delle vittime Lucane, Piemontesi e Lombarde; in effetti l'accaduto è stato riferito dalla metà delle vittime Lombarde, da un terzo di quelle della Basilicata e da una su cinque del Piemonte. Il tempo necessario è stato non inferiore all'anno, ma in alcuni casi, per esempio nello studio di Varese è emerso che il tempo impiegato è stato di quattro anni. Nessuna delle vittime dei più recenti studi ha ricevuto un aiuto specifico da parte di un esperto mentre dalle ricerche lombarde è emerso che alcuni ragazzi (il 17\% ed il 9\%) è stato aiutato da personale esperto.

\section{Valutazione complessiva dell'incidenza dell'abuso}

La ricerca ha mostrato come per le varie tipologie di abuso indagate vi sia una diversa prevalenza di abusati, mettendo in luce quanto la definizione di abuso influisca sulla valutazione della prevalenza.

Considerando tutte le forme di abuso indagate, abbiamo rilevato nello studio Piemontese 50 casi, pari al $12,9 \%$ del campione, suddivisi in 27 nei maschi $(13,1 \%)$ e 23 femmine $(12,6 \%)$ mentre in quello Lucano i casi emersi sono stati 42 , pari al $34,1 \%$ del campione, suddivisi in 34 maschi $(30,8 \%)$ e 8 femmine $(6,5 \%)$ mentre una vittima non ha specificato il sesso. I casi reali potrebbero però essere ancora di più (64, pari al $16,4 \%$ per il primo studio e 53 , pari al $43 \%$ per il secondo), se, come è stato fatto in altre ricerche, vengono considerate vittime di abuso non solo coloro che lo dichiarano ma anche coloro che si nascondono dietro la risposta preferisco non rispondere. Nelle precedenti ricerche si sono rilevati 415 casi, pari al 14,6\% (questo dato potrebbe salire a $15,4 \%$ se venissero considerati gli studenti che hanno preferito non rispondere) divisi in 318 femmine $(14,6 \%)$ e 97 maschi $(12,5 \%)$ a Milano, mentre a Varese sono stati individuati 70 casi, pari al $12,5 \%$ del campione, suddivisi in 22 nei maschi (10,5\%) e 48 femmine (13,7\%). Quanto sopra descritto è illustrato nella Figura 6. La prevalenza di abuso sessuale nei campioni indagati è pertanto compresa tra $12,5 \%$ (Varese) e 34,1\% (Lauria): l'analisi mostra una prevalenza complessiva, in Basilicata, decisamente superiore rispetto a quanto emerso nelle precedenti ricerche da un lato e dall'altro appare una maggior frequenza di vittime di sesso maschile per tutte le tipologie di abuso indagate ad eccezione dell'essere toccati in parti intime. Nei restanti studi si è rilevata una prevalenza complessivamente superiore nelle femmine ad eccezione dell'esposizione a materiale pornografico.

La Tabella 1 sottostante illustra la classificazione degli abusi in lievimoderati e gravi per tutte le ricerche: si osserva che molti dei casi riportati rientrano nelle prime tre categorie di abuso, visione di materiale pornografico e toccato o costretto a toccare parti intime e che nelle ricerche di Milano, Varese e Piemonte i risultati sono sovrapponibili. Tuttavia, si deve sottolineare il picco relativo sia agli abusi lievi-

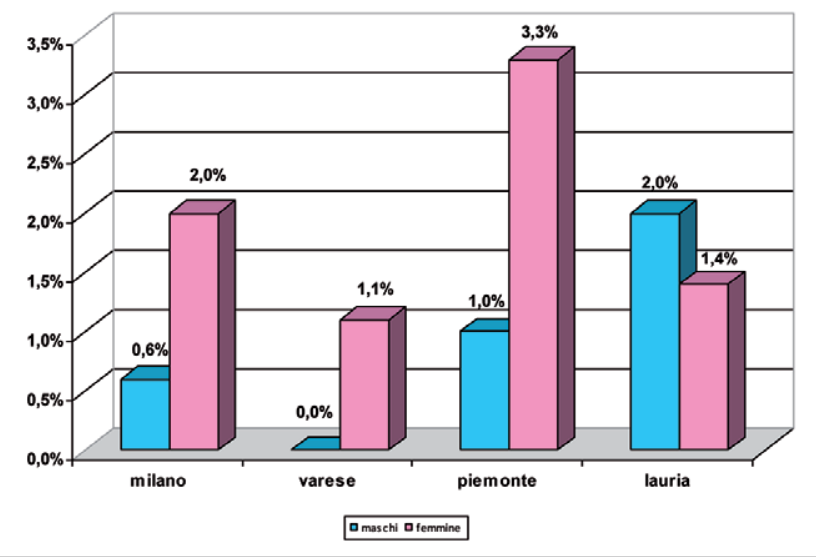

Figura 4. Essere costretto a masturbare un altro/a.

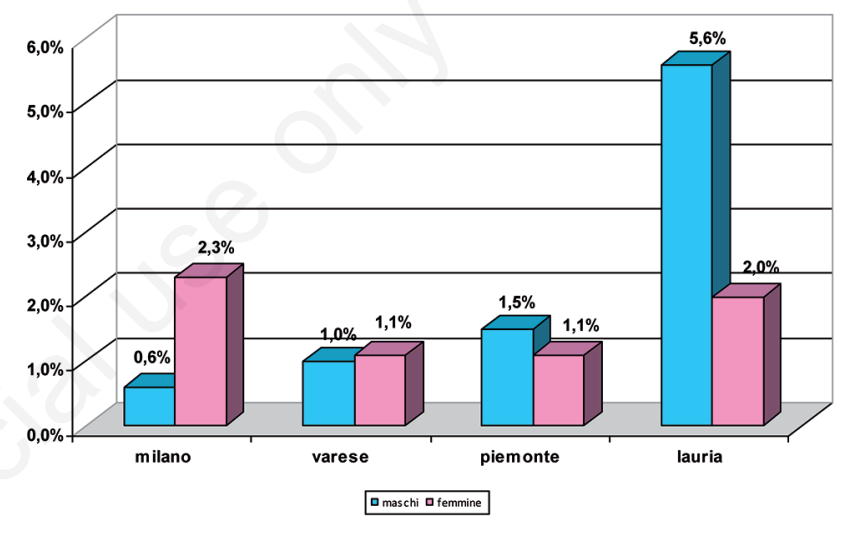

Figura 5. Essere stati penetrati.

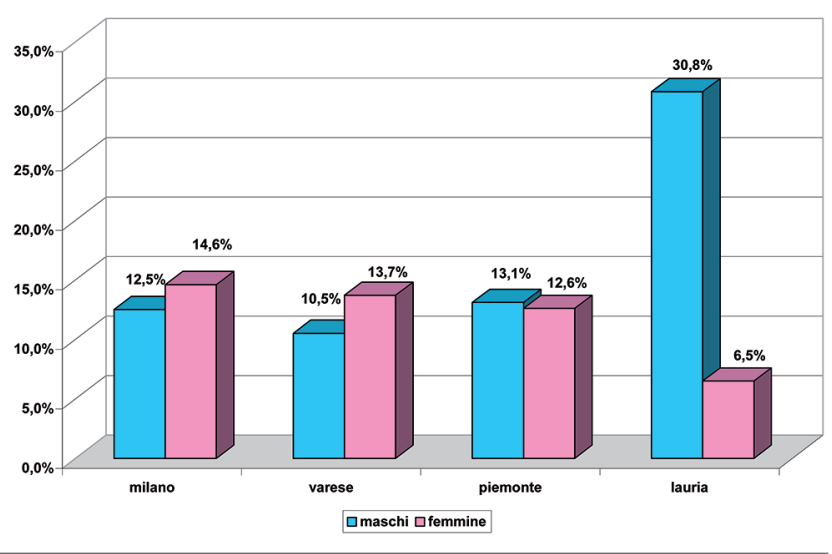

Figura 6. Incidenza di tutti i tipi di abuso.

Tabella 1. Tasso di prevalenza rilevato nei quattro territori indagati.

\begin{tabular}{lcccc} 
& Milano & Varese & Piemonte & Lauria \\
Abuso lieve-moderato & $11,80 \%$ & $10,70 \%$ & $10,30 \%$ & $21,10 \%$ \\
Abuso grave & $1,80 \%$ & $1,80 \%$ & $2,50 \%$ & $6,50 \%$ \\
\hline
\end{tabular}


moderati che gravi riscontrato in Basilicata: apparentemente nulla riesce a spiegare tali dati, anche se certamente ulteriori ricerche con campioni più numerosi del nostro andrebbero implementati al fine di verificare l'attendibilità dei nostri risultati.

Relativamente all'età in cui si è verificato il primo episodio nelle ricerche di Milano e Varese si è rilevato che tende a crescere con la gravità dell'abuso ed è, per ciascuna tipologia, più alta nei maschi rispetto alle femmine; nello studio Piemontese si mantiene piuttosto costante e non aumenta con l'aumentare della gravità dell'abuso e risulta superiore nei maschi rispetto alle femmine. Infine, per quanto riguarda la Basilicata, l'età media in cui è stato subito il primo episodio di abuso sembra, per i maschi essere più alta per le tipologie di abuso considerate lievi ed è per ciascuna tipologia, più alta nei maschi rispetto alle femmine.

Spesso l'abusante è ben conosciuto dall'abusato, aspetto che risulta vero sia per le vittime di sesso femminile che per quelle di sesso maschile. In molti casi si tratta di un membro appartenente alla famiglia allargata, come per esempio zio, cugino; tuttavia non è trascurabile il numero delle vittime che ha riportato di aver subito abuso da più persone. Inoltre, negli studi Lombardi è piuttosto consistente anche il numero di vittime che ha riportato un abuso dal partner 0 ex partner, dato peraltro non confermato dalle successive indagini.

\section{Conclusioni}

Alla luce di quanto sopra esposto, possiamo concludere che i risultati emersi nel corso del primo studio epidemiologico condotto a Milano, ormai alcuni anni orsono, sono stati ampiamente confermati dalle successive ricerche. I dati esposti sono chiari: tutte le tipologie di abuso indagate sono riferite dagli studenti che hanno partecipato alle ricerche, sia che si tratti di grandi città metropolitane che di piccole realtà provinciali. Quanto descritto in precedenza dovrebbe supportare ulteriormente da un lato la conferma della diffusione del fenomeno in tutte le realtà italiane, anche nel sottogruppo dei maschi, e dall'altro la necessità di avviare capillarmente in tutte le scuole del territorio programmi di prevenzione primaria dell'abuso sessuale da inserire nei curricula scolastici. Poiché l'età media in cui la vittima subisce l'abuso varia tra 9 e 15 anni si rafforza anche la convinzione che tali programmi devono essere proposti nelle scuole elementari, come è stato fatto per anni nella città di Milano, che per prima ha implementato il progetto Le parole non dette.

A proposito della ricerca effettuata in Basilicata possiamo affermare che questa è forse la prima ricerca puntuale che tenta di indagare il fenomeno anche nelle regioni del Sud. È possibile che il fenomeno sia ancora più sommerso e il livello di omertà più accentuato rispetto al Nord. Tuttavia, il nostro campione è davvero troppo piccolo, basti pensare che è pari ad un terzo degli studenti indagati in Piemonte, un quarto di quelli di Varese ed è 23 volte più piccolo del gruppo di Milano, per poter generalizzare i risultati e ulteriori ricerche devono essere condotte per confermare i risultati ottenuti.

\section{Bibliografia}

1. Finkelhor D. The international epidemiology of child sexual abuse. Child Abuse Neglect 1994;18:409-17.

2. Finkelhor D, Hotaling G, Lewis IA, Smith C. Sexual abuse in a national survey of adult men and women: prevalence, characteristics and risk factors. Child Abuse Neglect 1990;14:19-28.

3. Krugman S, Mata L, Krugman R. Sexual abuse and corporal punishement during childhood: a pilot retrospective survey of university students in Costa Rica. Pediatrics 1992;90:157-61.

4. Ruiz ZA. Abuso sexual al menor en Republica Dominicana. In: Proc. Sociedad Internacional de Pediatria "Abuso, abandono y violencia al nino: una perspectiva del Caribe y America Latina". Dominican Republic; 1990.

5. Lopez F. Abusos sexuales de menores. Salamanca, Spain: Ministerio de Asuntes Sociales y Universidad de Salamanca; 1992.

6. Agathonos H, Alexandridis A, Fereti I. A retrospective study of child abuse among Greek university students: implications for prevention and future research. In: Proc 9th Int Congr on Child and Abuse. Chicago, IL, USA; 1992.

7. Levett JF, Biebl WG. Childhood sexual abuse: a study of prevalence in a non clinical sample in Austria. Innsbruck University Department of Psychiatry; 1989.

8. Muhammad M, Haj-Yahia, Safa T. The rates of child sexual abuse and its psychological consequences as revealed by a study among Palestinian university students. Child Abuse Neglect 2001;25: 1303-27.

9. Chen J, Dunne MP, Han P. Child sexual abuse in China: a study of adolescents in four provinces. Child Abuse Neglect 2004;28:1171-86.

10. Sandler J, Fonagy P. Recovered memories of abuse: true or false? London, UK: Karnac Books; 1997.

11. Pellai A, Bassoli L, Castelli B, et al. Risultati di uno studio di prevalenza su un campione di 3000 studenti frequentanti le classi $\mathrm{V}$ delle scuole superiori della città di Milano. Maltrattamento e Abuso all'Infanzia 2004;6:79-96.

12. Zerilli M, Rigoni S, Caldana L, et al. Ricerca epidemiologica sulla prevalenza dell'abuso sessuale in età evolutiva. Maltrattamento e Abuso all'Infanzia 2002;4:73-104.

13. www.sosinfanzia.it

14. Bianchi D, Moretti E. Vite in bilico. Indagine retrospettiva su maltrattamenti e abusi in età infantile. Firenze, Italy: Istituto degli innocenti; 2006. 